\title{
EXPLORATORY FIELD TRIAL OF MOTORCYCLE AUTONOMOUS EMERGENCY BRAKING (MAEB): CONSIDERATIONS ON THE ACCEPTABILITY OF UNEXPECTED AUTOMATIC DECELERATIONS
}

\author{
AUTHORS \\ Fitzharris $^{\mathrm{b}}$, Michael G. Lennéb \\ AFFILIATIONS \\ a Department of Industrial Engineering, University of Florence \\ Via di Santa Marta 3, 50139 Firenze, Italy \\ ${ }^{\mathrm{b}}$ Monash University Accident Research Centre, Monash University \\ 21 Alliance Lane - Monash University, Clayton Campus, Victoria 3800 Australia
}

Giovanni Savino ${ }^{\mathrm{a}, \mathrm{b}}$ (corresponding author: giovanni.savino@unifi.it), Marco Pierini ${ }^{\mathrm{a}}$, Jason Thompson ${ }^{\mathrm{b}}$, Michael

This is an Accepted Author Version of an original article submitted for consideration in Traffic Injury Prevention (copyright Taylor \& Francis) and accepted for publication on 14 February 2016. The article will be available online at: http://dx.doi.org/10.1080/15389588.2016.1155210

\begin{abstract}
Objective. Autonomous emergency braking (AEB) acts to slow down a vehicle when an unavoidable impending collision is detected. In addition to documented benefits when applied to passenger cars, AEB has also shown potential when applied to motorcycles (MAEB). However, the feasibility of MAEB as practically applied to motorcycles in the real world is not well understood.

Methods. In this study we performed a field trial involving 16 riders on a test motorcycle subjected to automatic decelerations, thus simulating MAEB activation. The tests were conducted along a rectilinear path at nominal speed of $40 \mathrm{~km} / \mathrm{h}$ and with mean deceleration of $0.15 \mathrm{~g}$ (15\% of full braking) deployed at random times. Riders were also exposed to one final undeclared brake activation with the aim of providing genuinely unexpected automatic braking events.

Results. Participants were consistently able to manage automatic decelerations of the vehicle with minor to moderate effort. Results of undeclared activations were consistent with those of standard runs.

Conclusions. This study demonstrated the feasibility of a moderate automatic deceleration in a scenario of motorcycle travelling in a straight path, supporting the notion that the application of AEB on motorcycles is practicable. Furthermore, the proposed field trial can be used as a reference for future regulation or consumer tests in order to address safety and acceptability of unexpected automatic decelerations on a motorcycle.
\end{abstract}

\section{KEYWORDS}

Motorcyclist, collision mitigation, autonomous emergency braking, tests, acceptability.

\section{INTRODUCTION}

Around the world, the use of motorcycles and other powered-two-wheelers (PTWs) for purposes of commuting continues to increase rapidly (Kopp 2011). The smaller size and manoeuvrability of motorcycles in dense or congested traffic provide them with relative speed and convenience advantages over larger vehicles such as cars, trucks, and buses (Lee, Polak et al. 2009). Manoeuvrability, flexibility, and control of PTWs is therefore a central attraction of riders toward PTW use over other transport modes (Chang and Wu 2008). However, the characteristics that make PTWs attractive also make it challenging to develop acceptable safety measures to avoid conflicts with other road users and to protect the passengers in case of collision.

Research has demonstrated that PTW users and their passengers are at significantly increased risk of death and injury per $\mathrm{km}$ travelled than other motorised road users (e.g., Beck, Dellinger et al. 2007). The risks for motorcyclists arise as a consequence of several factors: the potentially high-speed of PTWs (Yannis, Antoniou et al. 2012); the lack of protection for riders in the event of a crash (Keall and Newstead 2012); the demanding control of PTWs under challenging road (e.g., slippery or rough surfaces) or traffic conditions (e.g., braking or swerving to avoid a collision), and the low conspicuity of PTWs (Crundall, Crundall et al. 2012, Gould, Poulter et al. 2012, Mitsopoulos-Rubens and Lenne 2012) .

Multi-vehicle crashes caused by failures of either the driver or rider make up a significant proportion of incidents involving PTWs (Penumaka, Savino et al. 2014). For example, in the MAIDS database (MAIDS 2009), multivehicle crashes involving PTWs caused by human errors accounted for $37 \%$ of the sample. In a third of these cases the rider did not attempt any avoidance or braking manoeuvre at all (Penumaka, Savino et al. 2014) therefore failing to reduce impact speed.

The impact speed of a vehicle at the point of collision is a major determinant of crash severity - especially among vulnerable road users. The likelihood of death and serious injury for vulnerable road users such as pedestrians, cyclists and other persons with low levels of external protection from injury is non-linear and increases 
significantly at speeds greater than $30 \mathrm{~km} / \mathrm{h}$ (Davis 2001). Therefore, in addition to reducing the incidence of collisions and increasing the protection afforded to PTW riders through the use of helmets or other protective clothing (Fitzharris, Dandona et al. 2009, de Rome, Ivers et al. 2012), reducing speed at the point of impact in the event of a crash is another means by which injuries or deaths associated with PTWs may be reduced. Attempts to reduce impact speeds have traditionally been approached from a macro perspective through the enforcement of speed limitations and traffic-calming measures (e.g., narrowing of roads, speed bumps, etc.) (Newstead, Hoareau et al. 2002, Bunn, Collier et al. 2003, Cameron and Delaney 2006, Victorian Auditor General 2006), or with intelligent speed adaptation systems (Carsten and Tate 2005). Another possible approach is to intervene with a micro perspective extending principles of impact speed reduction in the likelihood of an inevitable or impending crash. A prominent example of this approach is Autonomous Emergency Braking (AEB), which has been successfully introduced to passenger vehicles (Rizzi, Kullgren et al. 2014). AEB uses a combination of sensors to judge the likelihood of impending collisions in the pre-crash phase. The system then applies a braking mechanism to either prevent or reduce impact speed (Rosén 2013).

In comparison to their widespread adoption within passenger vehicles (Hulshof, Knight et al. 2013) the application of AEB systems in motorcycles (MAEB) has lagged, with no applications currently available in the consumer market. This situation persists despite the following two facts. First, autonomous emergency braking was identified as a priority area of research for the European Powered Two-wheeler Integrated Safety Project (PISa) (Savino, Pierini et al. 2010). And second, recent accident reconstruction, modelling, and 'laboratory' evidence (Savino, Pierini et al. 2012, Symeonidis, Kavadarli et al. 2012) (using mild decelerations within controlled test environments) showed MAEB's potential to mitigate PTW crash severity (Savino, Giovannini et al. 2013, Savino, Pierini et al. 2013). This lack of attention given by the research community on MAEB is perhaps also surprising given the widespread integration and known benefit of antilock braking systems (ABS) within the PTW fleet (Rizzi, Strandroth et al. 2009, Roll, Hoffmann et al. 2009, Teoh 2011, Savino, Pierini et al. 2013), and existing examples of integration of stability control systems on PTWs (Matschl 2014).

Whilst little confirmatory evidence exists at this stage, a potential hindrance to consumer demand-driven development of MAEB may be acceptability of assistive systems among riders. Previous studies have identified barriers to acceptability of rider assistance systems including perceived usability/satisfaction, usefulness, effectiveness, and affordability (Beanland, Lenné et al. 2013). Beanland et al. (2013) found that levels of acceptance for rider assistance systems are often lower than equivalent systems for passenger cars, due to a common intention among riders of keeping full control of their own vehicle. With this in mind, even if we assume comparable attributes between AEB and MAEB such as usefulness, effectiveness and affordability, acceptance of MAEB among riders cannot be guaranteed; hence, usability still needs to be demonstrated. The usability of MAEB systems that supplement, or remove, rider control may be unacceptable to end-users. Some aspects of the system design can assist to overcome these barriers, these being: i) if MAEB is designed to enhance rider safety within an inevitable crash circumstance (Savino, Giovannini et al. 2016) that is unlikely to be the rider's fault (Schneider, Savolainen et al. 2012), and ii) MAEB is demonstrated to be potentially effective (Savino, Rizzi et al. 2014). Thus, testing system usability and acceptability is important if safety benefits of MAEB technology are to be realised. Initial experiments on MAEB usability have been described in the literature. In a study conducted in controlled laboratory conditions, the effect of sudden decelerations among PTW riders in 'pre-warned' and 'no-warning' conditions were analysed to identify an upper limit for deceleration values below which forward displacement of the rider is negligible (Symeonidis, Kavadarli et al. 2012). Whilst instructive, the deceleration was produced by means of a sled mimicking the riding position, and hence neglected the control task that riders must perform during deceleration of a real PTW.

Alternatively, Savino, Pierini et al. (2012) described a test track trial with participants riding a scooter toward a foam obstacle without braking, until an MAEB system deployed an automatic deceleration. The study reported good rider acceptance of the system and generally confirmed the deceleration values recommended by Symeonidis et al. (2012). However, riders were primed to expect MAEB deployment through observation of the obstacle getting closer and therefore may have adjusted their riding posture in anticipation. Furthermore, participants were professional test riders rather than 'typical'.

Little evidence is available concerning the feasibility of automatic braking events for non-alerted riders. It could be argued that automatic braking events need to be truly unexpected in order to test MAEB usability in real-worldlike conditions, that is, when the user may not be aware of an imminent collision that triggers autonomous deceleration. Furthermore, quantitative measurements, including physiological responses such as heart rate (Mehler, Reimer et al. 2009), would help to further understand participants' subjective responses.

In this study, we aimed to evaluate the response of riders when subjected to automatic braking events activated at a random time, simulating the effects of MAEB activations. As far as the authors are aware, this is the first time that non-professional riders have tested automatic braking in a field trial. In particular, we attempted to reproduce genuine unexpected braking events within a controlled test environment to observe the behaviour of participants and obtain riders' subjective evaluation of the MAEB system's acceptability. Physiological response and effects of the deceleration on post deployment vehicle control were also considered. 


\section{METHOD}

\section{Participants}

Ethics approval for this study was granted by the Monash University Human Research Ethics Committee, Victoria, Australia (Project N. CF13/2749 - 2013001475).

Criteria for participation were as follows: a) hold a valid, full motorcycle licence (Australian class R) with no restrictions; b) ride at least once per week, and c) not be under temporary or permanent medical conditions representing contraindications to safe riding (e.g. intoxication). Participation involved attending a test track area for a single two-hour session with a reimbursement of $\$ 30$.

A pool of fifty-eight potential participants were initially identified through a combination of: a) an existing database of participants from previous road safety studies (37); b) via University newsletter (8); c) among colleagues (3), and d) others (10). The response rate was 62\%. Two respondents were no longer active motorcyclists anymore, 3 people refused the invitation, 15 people initially accepted but dropped out before the test. In total, 16 participants completed the study (15 male, 1 female). The characteristics of this sample are presented in Table 1.

The age of participants ranged between 20 and 60 years. All participants but one owned at least one motorcycle at the time of the test. The most frequent type of motorcycle owned by the participants was a sports bike. Just over half the participants ( 9 riders) declared their motorcycle to be their main means of transportation. The majority of participants $(n=10)$ indicated they rode mainly for commuting purposes $(n=6)$, and reported riding more than once a week. Six participants reported daily usage. Distance travelled by participants ranged from less than 1,000 $\mathrm{km}$ to more than 20,000 km per year. Participants had held a motorcycle licence for a minimum of 4 years to a maximum of 36 years. Every participant also owned a car licence, and four participants owned additional heavy vehicle licences (Australian Light Rigid (LR) or Medium Rigid (MR) categories). Nine participants had taken part in at least one non-mandatory rider course in the past, including five out of the six sports bike owners. Further participant details are provided in the Appendix.

\section{Test Vehicle and Equipment}

The test vehicle was a Honda CBR125R MY 2011, a light sports style motorcycle equipped with a four-stroke electronically injected single cylinder engine. General characteristics of the test vehicle are given in Table 2.

The test vehicle was equipped with a custom-made, remote controlled braking device that allowed the investigator to produce an automatic deceleration of the test vehicle at a desired time. The automatic braking device $(A B)$ operated by turning off the engine. This design solution is simple, low-cost, reliable, repeatable and safe, as the deceleration values it produces are intrinsically limited. The drawback is that the deceleration cannot be adjusted nor modulated during the automatic braking event. When engines such as that equipping the test vehicle are turned off, the positive motor torque produced during the power stroke is null and the engine torque acting on the crankshaft is chiefly the negative torque produced during the intake, compression and exhaust strokes executed on fresh air. This negative engine torque is applied to the rear wheel via the transmission system and acts as a selflimited braking torque. The intensity of the engine braking torque is a monotonic function of the engine angular speed. Given a vehicle speed, the engine braking torque is maximised by engaging the lowest transmission ratio $\tau=\frac{\omega_{r}}{\omega_{e}}$, where $\omega_{\mathrm{r}}$ and $\omega_{\mathrm{e}}$ are the angular speeds of the rear wheel and the engine crankshaft, respectively. Consequently, the engine braking torque is higher with lower gears. A typical range of deceleration produced by the engine braking force in first gear is from $1 \mathrm{~m} / \mathrm{s}^{2}$ to $3 \mathrm{~m} / \mathrm{s}^{2}(0.1 \mathrm{~g}$ to $0.3 \mathrm{~g}$, where $g$ is the acceleration of gravity), corresponding to $10 \%$ to $30 \%$ of the deceleration produced during full braking manoeuvres in typical conditions on dry asphalt.

The test vehicle was also equipped with: a) two cameras mounted on the windshield (backward-facing towards the rider) and on the petrol tank (forward-facing); b) a signal converter (based on a PEAK PCAN-MicroMod); c) a data logger with Global Positioning System (GPS) providing vehicle position, speed, and longitudinal and lateral accelerations (Video VBOX Lite), and d) a triaxial accelerometer (via smartphone sensors logged with a basic data logging software). The signal converter was responsible for encoding on-board digital and analogic signals into a standard Controller Area Network (CAN) bus message recorded via data logger (front and rear brake activation, clutch position, throttle position, and automatic deceleration device status). The data logger performed data collection at a sample rate of $10 \mathrm{~Hz}$, synchronising vehicle signals and videos from the on-board cameras. Data points were time stamped with high precision GPS time. Throttle position was also marked to be visible in the video footage.

A validation of the travelling speed was performed in post processing via integration of the GPS measured speed along the activation stretch $(80 \mathrm{~m})$. This validation was conducted using five runs without $\mathrm{AB}$ activation, randomly chosen among different riders. Results indicated that error in speed measurement at approximately constant speed was lower than one percent.

GPS-based acceleration was computed as mean acceleration of the GPS speed data between a given time interval. Acceleration and rotation data measured via smartphone sensors were filtered with moving average filter, using a symmetric time window of \pm 7 samples. 
Throttle measurements used voltage signal from the throttle position sensor connected to the motorcycle ECU. At the time of vehicle set up, the throttle measurement electrically interfered with the ECU. The throttle sensor was eventually disconnected to guarantee correct functioning of the engine during the tests. Qualitative analysis of the throttle position was still possible via on-board video recorded data.

A Zephyr BioHarness device worn on the participant's chest recorded heart rate and acceleration data during all the phases of the experiment (sample rate of $1 \mathrm{~Hz}$ ). The list of measurements is shown in Table A1 in the Appendix. Procedure

The tests were conducted on a dedicate test track in Victoria, Australia. Each test consisted of three phases in a single 90 minute session.

Test phase I: Participants received an explanatory statement presenting an overview of the test procedure and risks involved, and provided informed consent. Prior to any riding tasks participants were asked to wear the BioHarness sensor to monitor heart rate and to wear full motorcycle protective gear for safety during the tests. A questionnaire was then completed to obtain demographic data and details of participants' relevant riding histories. Test phase II: Before beginning the test runs, participants were provided with ten minutes of 'free riding' time within the test circuit to familiarise themselves with the vehicle. Participants were then provided with instructions regarding the test protocol as detailed hereafter. In each test run, participants accelerated the vehicle from a standing start in first gear up to a nominal speed of $40 \mathrm{~km} / \mathrm{h}$ as judged by the speedometer to be reached before passing through a virtual gate $30 \mathrm{~m}$ off the start, marked with street cones (acceleration stretch). The actual speed was somewhat below the nominal speed due to the typical overestimated measurement of speedometers. Participants usually maintained full throttle during the test run as the vehicle automatically stabilised at a speed of approximately $40 \mathrm{~km} / \mathrm{h}$ of the speedometer. This was due to an inbuilt limitation in the engine revolutions speed operated by the standard electronic control unit (ECU) of the vehicle. This procedure had the advantage of removing additional mental load associated with tracking speed. Participants then entered an $80 \mathrm{~m}$ stretch in which automatic deceleration may or may not have taken place (activation stretch). During runs involving automatic deceleration events, these were remotely triggered by the investigator at nominal discrete random times of $1 \mathrm{~s}, 2$ $\mathrm{s}$, and $3 \mathrm{~s}$ after the vehicle entered the activation stretch, with counterbalanced presentation sequence among participants. Participants were not informed about the existence of nominal activation times. Participants were instructed to let the vehicle cruise after automatic deceleration (i.e. without controlling brakes, clutch, or throttle) and to safely stop the motorcycle using standard brakes when reaching a speed below $5 \mathrm{~km} / \mathrm{h}$ or if the vehicle became unstable. If the activation did not take place during a run, participants reached a second gate where they were instructed to apply manual brakes and come to a complete stop (final stretch).

The test included two initial sets of twelve runs with a 5-minute rest break in between. In attempts to manage participant expectancy approximately $50 \%$ of runs involved the automatic deceleration event. The tests also included a final run designed to create a scenario in which participants did not expect an activation of the automatic deceleration. For this, at the end of the second set riders were told that they would have performed an additional set of five baseline runs without any activation of the automatic deceleration. However, during the first run automatic braking was triggered in the activation stretch (refer to Figure 1). In the remainder of the paper, these events will be referred to as 'undeclared activations' in order to differentiate them from 'standard activations' associated with set one and two.

The second phase was video recorded using a fixed camera positioned behind the starting point. The investigator who was activating the decelerations was positioned twelve meters aside the acceleration stretch, and seven meters before the start of the activation stretch.

Test phase III: Participants then removed the heart rate sensors, completed a questionnaire concerning their subjective evaluation of the test experience, and responded to a five-minute interview. In the questionnaire, participants provided their evaluation regarding: a) the effort needed to control the vehicle post $\mathrm{AB}$ deployment (with a marking on a vertical segment labelled with a scale from "absolutely no effort" to "extreme effort"); b) the levels of intensity and smoothness of the automatic deceleration (with a 7 level scale ranging from "much too low" to "much too high"), and c) an overall opinion of the system (with a 6 level scale ranging from "very bad" to "excellent"). The design of the questionnaire was informed by previous research investigating rider assistance systems using field trials (Pauwelussen, Oudenhuijzen et al. 2010). In the interview participants were asked their opinions about experiencing automatic decelerations and whether they felt the need to operate any action during the automatic deceleration events. Participants were also asked whether or not they anticipated automatic braking in the 'undeclared activation' events.

\section{Data Analysis}

The 'idle' state signal of the engine-stop device was used as a marker to identify the time frame of each run; the state change from 'idle' to 'active' indicated system deployment. The time when entering the activation stretch (time at gate) was identified via Matlab script using GPS data combined with engine-stop device state signal at start, given the length of the acceleration stretch. The automatic detection of the time at gate was validated using the on-board video recorded data. The gate cones were visible when passing by the gate (accuracy of $\pm 0.1 \mathrm{~s}$ ). 
Speeds at gate and at activation were identified, together with the time interval between gate and activation. Mean decelerations were computed on a $1.5 \mathrm{~s}$ time frame starting $0.5 \mathrm{~s}$ after activation of the automatic braking.

Script failures were reported in 29 test runs (7\% of the cases).

Brake and clutch signals, together with video recorded data, were used to check that participants did not apply any action within $2 \mathrm{~s}$ from the activation of the automatic deceleration.

Descriptive statistics were applied to the deceleration values across the participants. A t-Student test was used to check the consistency of the automatic deceleration events in standard and undeclared activations (Keppel 1991).

\section{RESULTS}

\section{Test Runs}

Each participant performed 24 to 26 runs plus the run involving undeclared activations. Participants experienced a percent of autonomous deceleration events ranging from $44 \%$ to $56 \%$ (except for the first participant P01, who was used as extra pilot tests for the system, experiencing $71 \%$ of activations). Participant P01 did not experience any undeclared activation. In total, 400 runs were performed, with 208 runs (52\%) involving autonomous deceleration.

Automatic decelerations were activated after mean time delays of $0.72 \mathrm{~s} \mathrm{(SD} \mathrm{0.19} \mathrm{s),} 1.61 \mathrm{~s}$ (SD 0.27 s), and 2.70 $\mathrm{s}(\mathrm{SD} 0.34 \mathrm{~s})$ after passing the gate, respectively for the $1 \mathrm{~s}, 2 \mathrm{~s}$, and $3 \mathrm{~s}$ nominal delay times. Frequencies of $30 \%$ for each nominal delay in each session were presented to each participant. In the tests, up to five consecutive 'no activation' cases were reported.

The mean speed at activation across all riders was $36.93 \mathrm{~km} / \mathrm{h}(\mathrm{SD} 0.49 \mathrm{~km} / \mathrm{h})$.

\section{Qualitative and Quantitative Analysis of the Automatic Decelerations}

The mean deceleration upon activation after the initial ramp was $1.46 \mathrm{~m} / \mathrm{s}^{2}\left(\mathrm{SD} 0.14 \mathrm{~m} / \mathrm{s}^{2}\right)$. Mean decelerations computed for each participant ranged from $1.21 \mathrm{~m} / \mathrm{s}^{2}$ to $1.57 \mathrm{~m} / \mathrm{s}^{2}$ (see Table 3). The variability in the decelerations is explained by the absence of a closed loop control of the deceleration in the AB and was reasonably affected by inter- and intra-participant factors such as body mass, size, and posture (influencing the system inertia and aerodynamics).

Despite the fact that average decelerations were observed to be in the lower part of the original design range (1 $\mathrm{m} / \mathrm{s}^{2}-3 \mathrm{~m} / \mathrm{s}^{2}$ ), acceleration measurements revealed peaks in the longitudinal deceleration of $3 \mathrm{~m} / \mathrm{s}^{2}$ (see example case depicted in Figure 2). The average deceleration over a time interval of $2 \mathrm{~s}$ of activation confirmed the values obtained with the GPS measurements, being less than $1.9 \mathrm{~m} / \mathrm{s}^{2}$ for all test runs.

\section{Analysis of Participants' Reactions}

Qualitative observations of videos from the test runs, both from on-board and external cameras (see Figure 3), did not highlight any significant loss of control caused by the activation of the automatic deceleration or by subsequent reactions of the participant. A sharp and small forward-leaning movement of the upper body (torso) was observed in the videos after automatic braking deployment, with typical duration of $0.5 \mathrm{~s}$. A small subsequent forward leaning of the head was also occasionally observed. Participants' arms appeared to remain steady. No visible movement on the handlebar was observed for most of the participants. In a few cases, however, a small control action on the handlebar was noticeable. A mild action of the rider on the throttle grip after activation was noticed in some of the cases. This action tended to slightly increase the throttle with duration of $0.2 \mathrm{~s}$ consistent with observations in cars (Kobiela and Engeln 2010).

\section{Key Heart Rate Results}

As riders approached the gate, there was an indication of anticipating the deployment of automatic deceleration, as seen through increased heart rate. Importantly, there was no evidence of a distinct increase in the heart rate after deployment of automatic decelerations. Concerning the runs involving undeclared activation events, qualitative analysis showed that heart rates were similar to those of standard runs. A fuller explanation of the heart rate analysis is described in the Appendix.

\section{Subjective Evaluations}

Participants provided their feedback on the system using a self-report questionnaire at the end of the test session. In the questionnaire, the item regarding the smoothness of the automatic deceleration resulted in some degree of ambiguity (i.e., a 'high smoothness' rating could have been interpreted either as 'too smooth' or 'too rough'). This issue was solved using participants' open-text comments to determine the direction of the scale. Ratings for smoothness were recoded as 'too smooth, 'perfect smoothness', or 'too rough'. Figure 4 shows a schematic of opinions sought by the study investigators. Results of the subjective evaluation are briefly reported in Table A2 in the Appendix.

Assuming a scale from 0 to 10 , representing 'absolutely no effort' (0) to 'extreme effort' (10), participants considered the effort needed to control the vehicle during automatic braking as low (2.2, SD 1.3, median value 2.5) on. Participants' overall opinion of the system converted in a scale from 0 ('very bad') to 10 ('excellent') was 6.0 (SD 2.1, median 6).

The majority of the participants $(n=10 ; 62 \%)$ declared that immediately after the activation of the automatic braking they felt the impulse to brake manually. This resulted in two participants slightly controlling the front 
brake shortly after the automatic deceleration in three runs each (P01 and P07). One participant actuated the clutch for a very short time just after the automatic deceleration in two occasions (P11). Five participants (31\%) reported an impulse to use the clutch however they were able to avoid engaging the clutch during automatic braking. One of them actually pulled the clutch during the automatic deceleration event in two occasions (P10). Four participants $(25 \%)$ declared that they felt no compulsion to operate any action in the initial phase after the automatic braking. These results have implications on the design, acceptability and potential effectiveness of the system, and warrant further investigation. Additional details of the comments provided by the participants are provided in Table A4 in the Appendix.

\section{Undeclared Activations}

Fifteen riders experienced a final test run with undeclared activation. During the interview, participants stated that this last activation was not anticipated. However, they declared it was not different from other activations. One stated that he suspected this last activation might occur. One participant stated that this last undeclared activation was more 'alerting' than in the previous runs, and 'looked at the gauges' thinking that 'something was wrong with the bike'. Interestingly, three participants suspected that the automatic deceleration during the undeclared activation was slightly more intense.

The average speed at the undeclared activation was $37.19 \mathrm{~km} / \mathrm{h}(\mathrm{SD} 0.37 \mathrm{~km} / \mathrm{h})$, and the average deceleration was $1.44 \mathrm{~m} / \mathrm{s}^{2}$ (SD $0.10 \mathrm{~m} / \mathrm{s}^{2}$ ) (see Table 3 for the deceleration values of each participant). The deceleration values during undeclared activations were similar to those of standard activations (t-Student test, $p=0.74$ ).

\section{DISCUSSION}

In this exploratory field trial, sixteen riders experienced automatic deceleration events on a real motorcycle when travelling along a straight path. Consistent with earlier studies examining MAEB, our results demonstrated that mild, automatic decelerations of a PTW as might be produced by an MAEB system can be easily managed by PTW riders. These results provide a level of confidence that MAEB systems producing mild decelerations do not adversely affect rider stability.

Small movements induced in the participants by the automatic deceleration were noticed: leaning torso, flipping head, and rotating hand. The stiff behaviour noticed in one of the riders (very limited movements of the torso) appeared to be associated with a slightly more evident rotation of the throttle handle-grip. The small sample involved in the present study, however, did not allow for detailed comparison of relative 'stiff' and 'floppy' participant behaviour. It is possible that at higher decelerations than those produced here the induced movements may increase, affecting both rider and PTW stability and control. In particular, possible impact on the throttle position unintentionally produced by the rider during higher decelerations should be further considered. In car AEB, preliminary warnings $0.90 \mathrm{~s}$ before automatic braking proved to be effective in reducing erroneous throttle controls at AEB deployment (Kobiela and Engeln 2010).

Participants' subjective evaluations revealed that limited effort was required to control the vehicle during the automatic deceleration events (including less experienced participants). Further, participants showed no discomfort with the intensity or smoothness of the deceleration, consistent with Symeonidis, Kavadarli et al. (2012) and Savino, Pierini et al. (2012).

Participants' statements about the final runs with undeclared activation suggested that during standard runs the automatic deceleration event may have been anticipated, rather than being genuinely unexpected. This may mean that the rider is better able to maintain control of the motorcycle than could be the case in the real-world where automatic deceleration is not expected. However, we found no relevant difference in the effects of automatic deceleration events between standard runs and those where automatic deceleration was not anticipated by the rider. Our interpretation is that the procedure used for standard activations can produce close representations of genuine unexpected automatic deceleration events as those in the undeclared activation runs.

Responses to questionnaires provided an overall positive evaluation of the specific system used in the tests. However, the interviews highlighted potential rider acceptability issues associated with MAEB. In particular, the ease to control the motorcycle after the automatic deceleration event was not always associated with a good overall opinion about the system. Other factors need to be addressed to achieve system acceptance. Importantly, of the six participants who were doubtful or concerned about the real-world application of MAEB before the tests, four reported more positive views of MAEB post-test after experiencing the system first-hand.

In conclusion, our findings suggested that a field trial involving repeated pseudo-unexpected decelerations is a viable way to investigate the influence of MAEB on the user, both from a functional and a safety perspective. In addition, this field trial enabled investigating rider acceptability of autonomous emergency braking for a motorcycle. The test protocol presented in this paper can be a reference for a future definition of standardised trials to be used either as regulation tests or as consumer tests that may contribute on system acceptability among the users.

\section{Limitations}

The small sample of motorcyclists recruited for this study limits the representativeness of the study findings. Despite the relatively wide range of ages, motivations for ridings, frequency of riding, experience, and riding style, 
we have no guarantee that participants were typical of the 'normal' PTW riding population. Additionally, the knowledge among participants that they were to be testing an MAEB system may have biased the sample toward riders for whom safety matters.

The tests analysed a limited range of manoeuvres (straight cruise at constant speed). The short duration of riding in each test likely contributed to the participants' expectancy of an event, despite the occurrence of deceleration events being randomised. A further limitation was that only one target speed and one target deceleration were tested. The target speed was chosen to reflect values where the estimated benefits of autonomous emergency braking are maximised, compatibly with the safety requirement of keeping the speed low (Savino, Giovannini et al. 2016), alongside safety considerations for early development stage tests. In Symeonidis et al. (2012), a value of $0.35 \mathrm{~g}$ for the autonomous deceleration did not induce additional instability in the participants compared to manually activated decelerations. However, these experiments were carried out on a sled and hence, did not involve vehicle rotations produced in a motorcycle during a braking manoeuvre (in particular pitching, and possibly also rolling and yawing). During our tests, no evident destabilisation of the vehicle and no control issue for participants was observed. This provided additional confidence that the implementation of MAEB systems is possible within PTWs. The present study confirmed that on a real, sports style motorcycle, no major instability of motorcyclists is induced when triggering an automatic deceleration of $0.15 \mathrm{~g}$ (mean value) along a straight path, starting from a constant speed of approximately $40 \mathrm{~km} / \mathrm{h}$.

Further investigations are required for proving the safety of autonomous braking on PTWs in real-life conditions. Longer duration of riding could be considered to reduce expectancy. More variables could be investigated, including the use of front/rear brakes instead of the engine brake, a range of decelerations and speeds at deployment, a range of manoeuvres (acceleration/braking and mild cornering), and the presence of other traffic.

\section{ACKNOWLEDGMENTS}

The research leading to these results has received funding from the European Union Seventh Framework Programme FP7/2007-2013 under grant agreement n. 328067 (ABRAM project).

The authors acknowledge Robert Bosch (Australia) Pty Ltd for supporting this study by providing the test track facility for the tests and by assisting in the logistics.

\section{ACRONYMS}

AB. Automatic braking

ABS. Antilock braking system

AEB. Autonomous emergency braking

CAN. Controller area network

GPS. Global positioning system

MAEB. Autonomous emergency braking for motorcycles

PTW. Powered two-wheeler

\section{REFERENCES}

Beanland, V., M. G. Lenné, E. Fuessl, M. Oberlader, S. Joshi, T. Bellet, A. Banet, L. Rößger, L. Leden, I. Spyropoulou, G. Yannis, H. Roebroeck, J. Carvalhais and G. Underwood (2013). "Acceptability of rider assistive systems for powered two-wheelers." Transportation Research Part F: Traffic Psychology and Behaviour 19: 6376.

Beck, L. F., A. M. Dellinger and M. E. O'Neil (2007). "Motor vehicle crash injury rates by mode of travel, United States: using exposure-based methods to quantify differences." American Journal of Epidemiology 166(2): 212218.

Bunn, F., T. Collier, C. Frost, K. Ker, I. Roberts and R. Wentz (2003). "Traffic calming for the prevention of road traffic injuries: systematic review and meta-analysis." Injury Prevention 9(3): 200-204.

Cameron, M. H. and A. Delaney (2006). Development of strategies for best practice in speed enforcement in Western Australia: Final Report. Report to the Department of the Premier and Cabinet. Office of Road Safety Western Australia. Melbourne, Monash University Accident Research Centre.

Carsten, O. M. J. and F. N. Tate (2005). "Intelligent speed adaptation: accident savings and cost-benefit analysis." Accident Analysis and Prevention 37(3): 407-416.

Chang, H.-L. and S.-C. Wu (2008). "Exploring the vehicle dependence behind mode choice: Evidence of motorcycle dependence in Taipei." Transportation Research Part A: Policy and Practice 42(2): 307-320.

Crundall, D., E. Crundall, D. Clarke and A. Shahar (2012). "Why do car drivers fail to give way to motorcycles at t-junctions?" Accident Analysis and Prevention 44(1): 88-96. 
Davis, G. (2001). "Relating Severity of Pedestrian Injury to Impact Speed in Vehicle-Pedestrian Crashes: Simple Threshold Model." Transportation Research Record: Journal of the Transportation Research Board 1773: 108113.

de Rome, L., R. Ivers, M. Fitzharris, N. Haworth, S. Heritier and D. Richardson (2012). "Effectiveness of motorcycle protective clothing: riders' health outcomes in the six months following a crash." Injury 43(12): 20352045.

Fitzharris, M., R. Dandona, G. A. Kumar and L. Dandona (2009). "Crash characteristics and patterns of injury among hospitalized motorised two-wheeled vehicle users in urban India." BMC Public Health 9(1): 11.

Gould, M., D. R. Poulter, S. Helman and J. P. Wann (2012). "Errors in judging the approach rate of motorcycles in nighttime conditions and the effect of an improved lighting configuration." Accidend Analysis and Prevention 45: 432-437.

Hulshof, W., I. Knight, A. Edwards, M. Avery and C. Grover (2013). Autonomous emergency braking test resutls. ESV Conference.

Keall, M. D. and S. Newstead (2012). "Analysis of factors that increase motorcycle rider risk compared to car driver risk." Accident Analysis and Prevention 49: 23-29.

Keppel, G. (1991). Design and analysis: A researcher's handbook, Prentice-Hall, Inc.

Kobiela, F. and A. Engeln (2010). "Autonomous emergency braking studies on driver behaviour." ATZ worldwide 112(10): 4-8.

Kopp, P. (2011). "The unpredicted rise of motorcycles: A cost benefit analysis." Transport Policy 18(4): 613-622.

Lee, T.-C., J. Polak and M. Bell (2009). "New Approach to Modeling Mixed Traffic Containing Motorcycles in Urban Areas." Transportation Research Record: Journal of the Transportation Research Board 2140: $195-205$.

MAIDS (2009). MAIDS: In-depth Investigations of Accidents Involving Powered two wheelers. Brussels, Belgium, ACEM.

Matschl, G. (2014). Motorcycle Sability Control - MSC. The next step into safety solutions for motorcycles. IFZ International Motorcycle Conference. Cologne.

Mehler, B., B. Reimer, J. Coughlin and J. Dusek (2009). "Impact of Incremental Increases in Cognitive Workload on Physiological Arousal and Performance in Young Adult Drivers." Transportation Research Record: Journal of the Transportation Research Board 2138: 6-12.

Mitsopoulos-Rubens, E. and M. G. Lenne (2012). "Issues in motorcycle sensory and cognitive conspicuity: the impact of motorcycle low-beam headlights and riding experience on drivers' decisions to turn across the path of a motorcycle." Accident Analysis and Prevention 49: 86-95.

Newstead, S., E. Hoareau and M. Cameron (2002). Evaluation of $50 \mathrm{~km} / \mathrm{h}$ speed limits in Victoria. Summary of interim analysis of all crashes and crashes involving pedestrians. Melbourne, Monash University Accident Research Centre.

Pauwelussen, J. J. A., A. J. K. Oudenhuijzen, N. J. M. Weijenberg and G. Savino (2010). Distance Support System For Rider Support During Car-Following. International Symposium on Advanced Vehicle Control AVEC10.

Penumaka, A. P., G. Savino, N. Baldanzini and M. Pierini (2014). "In-depth investigations of PTW-car accidents caused by human errors." Safety Science 68: 212-221.

Rizzi, M., A. Kullgren and C. Tingvall (2014). Injury crash reduction of low - speed Autonomous Emergency Braking (AEB) on passenger cars. $\underline{\text { IRCOBI } 2014 \text { Conference. }}$

Rizzi, M., J. Strandroth and C. Tingvall (2009). "The effectiveness of antilock brake systems on motorcycles in reducing real-life crashes and injuries." Traffic Injury Prevention 10(5): 479-487.

Roll, G., O. Hoffmann and J. Konig (2009). Effectiveness Evaluation of Antilock Brake Systems (ABS) for Motorcycles in Real-World Accident Scenarios. ESV Conference.

Rosén, E. (2013). Autonomous Emergency Braking for Vulnerable Road Users. IRCOBI 2013 Conference.

Savino, G., F. Giovannini, N. Baldanzini, M. Pierini and M. Rizzi (2013). "Assessing the Potential Benefits of the Motorcycle Autonomous Emergency Braking Using Detailed Crash Reconstructions." Traffic Injury Prevention 14(S1): S40-S49. 
Savino, G., F. Giovannini, M. Fitzharris and M. Pierini (2016). "Inevitable Collision States for Motorcycle-to-Car Collision Scenarios." IEEE Transactions on Intelligent Transportation Systems: 1-11 (in press).

Savino, G., M. Pierini and N. Baldanzini (2012). "Decision logic of an active braking system for powered two wheelers." Proceedings of the Institution of Mechanical Engineers, Part D: Journal of Automobile Engineering 226(8): 1026-1036.

Savino, G., M. Pierini, R. Grant, R. Frampton, R. Talbot, S. Peldschus, E. Schuller, A. Oudenhuijzen, J. Pauwelussen, B. Scheepers and A. Teerhuis (2010). PISa - Powered two-wheeler Integrated Safety. Development, implementation and testing of PTW integrated safety systems. IFZ International Motorcycle Conference, Cologne, Germany.

Savino, G., M. Pierini, M. Rizzi and R. Frampton (2013). "Evaluation of an autonomous braking system in realworld PTW crashes." Traffic Inj Prev 14(5): 532-543.

Savino, G., M. Pierini, M. Rizzi, R. Frampton and J. Thompson (2013). Can Experienced Riders Benefit from an Autonomous Emergency Braking System? International Motorcycle Safety Conference. Orlando, Florida, Motorcycle Safety Foundation.

Savino, G., M. Rizzi, J. Brown, S. Piantini, L. Meredith, B. Albanese, M. Pierini and M. Fitzharris (2014). "Further Development of Motorcycle Autonomous Emergency Braking (MAEB), What Can In-Depth Studies Tell Us? A Multinational Study." Traffic Injury Prevention 15(Sup1): S165-S172.

Schneider, W. H. t., P. T. Savolainen, D. Van Boxel and R. Beverley (2012). "Examination of factors determining fault in two-vehicle motorcycle crashes." Accident Analysis and Prevention 45: 669-676.

Symeonidis, I., G. Kavadarli, E. Shuller, M. Graw and S. Peldschus (2012). "Analysis of the stability of PTW riders in autonomous braking scenarios." Accident Analysis and Prevention 49: 212-222.

Teoh, E. R. (2011). "Effectiveness of antilock braking systems in reducing motorcycle fatal crash rates." Traffic Injury Prevention 12(2): 169-173.

Victorian Auditor General (2006). Making travel safer: Victoria's speed enforcement program, July 2006. Melbourne, State Government of Victoria.

Yannis, G., C. Antoniou, P. Evgenikos, P. Papantoniou and A. Kirk (2012). "Characteristics and Causes of Power Two Wheeler Accidents in Europe." Procedia - Social and Behavioral Sciences 48: 1535-1544. 
Table 1. Summary of the sample riders involved in the experimental activity

\begin{tabular}{lrr}
\hline & $\mathbf{n}$ & $\mathbf{\%}$ \\
\hline Age & & \\
$21-30$ & 4 & 25.0 \\
$31-40$ & 6 & 37.5 \\
$41-50$ & 3 & 18.7 \\
$51-60$ & 3 & 18.7 \\
unknown & 0 & 0.0 \\
Total & 16 & 100.0 \\
Type of bike owned & & \\
adventure & 4 & 25.0 \\
cruiser & 2 & 12.5 \\
sports & 6 & 37.5 \\
touring & 4 & 25.0 \\
unknown & 0 & 0.0 \\
Total & 16 & 100.0 \\
km travelled per year & & \\
$<1,000$ & 2 & 12.5 \\
1,000 - 5,000 & 3 & 18.7 \\
$5,000-10,000$ & 3 & 18.7 \\
$10,000-15,000$ & 3 & 18.7 \\
15,000 - 20,000 & 2 & 12.5 \\
$>$ 20,000 & 2 & 12.5 \\
unknown & 1 & 6.3 \\
Total & 16 & 100.0 \\
\hline & & \\
& &
\end{tabular}

Table 2. Characteristics of the test vehicle. $\uparrow$ Coordinates are referred to a SAE coordinate system originated in the point of contact between the rear wheel and the road plane (downward z-axis)

\begin{tabular}{ll}
\hline Property & Value \\
\hline Wheelbase & $1.31 \mathrm{~m}$ \\
Kerb weight & $137 \mathrm{~kg}$ \\
Seat coord. $(\mathrm{x}, \mathrm{z})^{\dagger}$ & $<0.44 \mathrm{~m},-0.80 \mathrm{~m}$ \\
Footpeg coord. $(\mathrm{x}, \mathrm{z})^{\dagger}$ & $0.39 \mathrm{~m},-0.27 \mathrm{~m}$ \\
${\text { Handlebar coord. }(\mathrm{x}, \mathrm{z})^{\dagger}}^{\dagger}$ & $0.92 \mathrm{~m},-0.92 \mathrm{~m}$ \\
\hline
\end{tabular}


Table 3. Number of runs, deceleration events, and mean decelerations values (minimum, maximum, mean, and during undeclared activation) for each participant. N.A.: not available

\begin{tabular}{lllllccc}
\hline Participant & $\begin{array}{c}\text { Runs } \\
\boldsymbol{n}\end{array}$ & $\begin{array}{c}\text { Activations } \\
\boldsymbol{n}\end{array}$ & $\begin{array}{c}\text { Min-max deceleration } \\
\text { for standard activations } \\
\left(\boldsymbol{m} / \mathbf{s}^{2}\right)\end{array}$ & $\begin{array}{c}\text { Mean deceleration } \\
\left(\boldsymbol{m} / \mathbf{s}^{2}\right)\end{array}$ & $\begin{array}{c}\text { SD } \\
\left(\boldsymbol{m} / \mathbf{s}^{2}\right)\end{array}$ & $\begin{array}{c}\text { Deceleration during } \\
\text { undeclared activation } \\
\left(\boldsymbol{m} / \mathbf{s}^{2}\right)\end{array}$ \\
\hline P01 & 24 & 17 & 1.37 & 1.65 & 1.53 & 0.09 & Not performed \\
P02 & 25 & 14 & 1.36 & 1.81 & 1.54 & 0.13 & 1.48 \\
P03 & 25 & 13 & 1.37 & 1.60 & 1.46 & 0.07 & 1.40 \\
P04 & 25 & 13 & 1.24 & 1.70 & 1.46 & 0.13 & 1.45 \\
P05 & 26 & 13 & 1.31 & 1.55 & 1.45 & 0.07 & 1.40 \\
P06 & 24 & 12 & 1.39 & 1.72 & 1.53 & 0.11 & 1.56 \\
P07 & 25 & 12 & 1.23 & 1.43 & 1.33 & 0.06 & N.A. \\
P08 & 25 & 13 & 1.30 & 1.66 & 1.48 & 0.11 & 1.47 \\
P09 & 25 & 13 & 1.21 & 1.60 & 1.44 & 0.12 & 1.48 \\
P10 & 26 & 13 & 1.08 & 1.39 & 1.20 & 0.10 & 1.39 \\
P11 & 25 & 13 & 1.44 & 1.85 & 1.57 & 0.13 & 1.52 \\
P12 & 25 & 12 & 1.15 & 1.58 & 1.34 & 0.11 & 1.15 \\
P13 & 25 & 11 & 1.21 & 1.68 & 1.43 & 0.14 & 1.52 \\
P14 & 25 & 13 & 1.34 & 1.74 & 1.51 & 0.13 & 1.51 \\
P15 & 25 & 13 & 1.28 & 1.69 & 1.48 & 0.12 & 1.48 \\
P16 & 25 & 13 & 1.31 & 1.63 & 1.45 & 0.09 & 1.35 \\
\hline
\end{tabular}

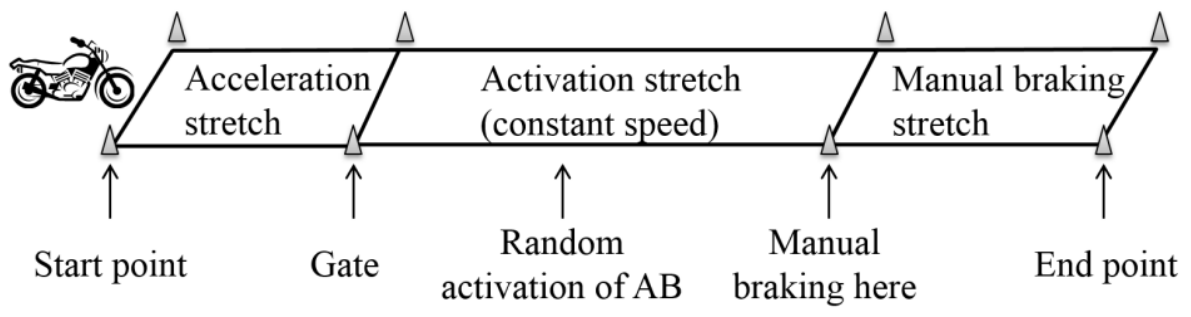

Figure 1. Scheme of the test track (not to scale)

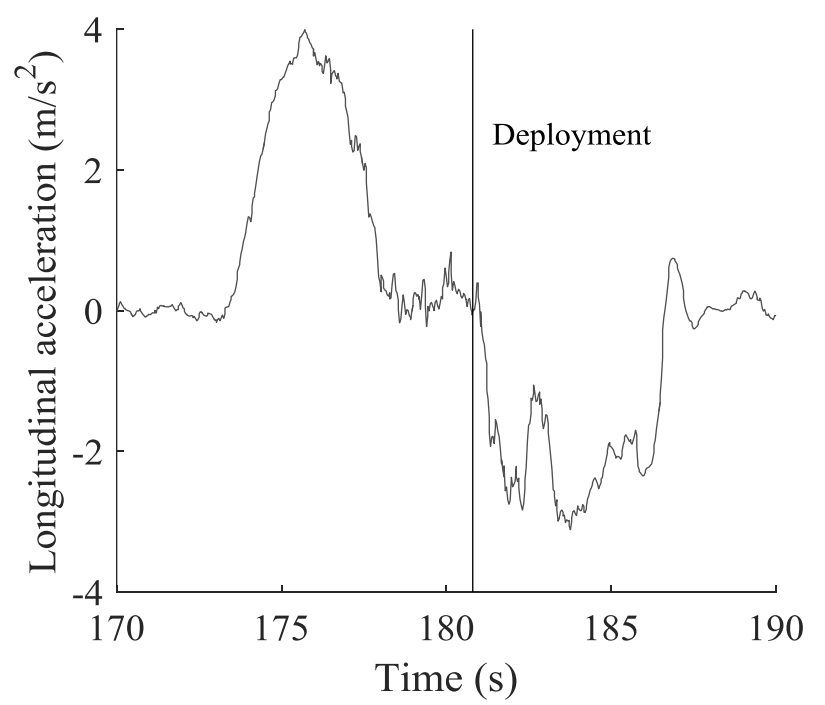

Figure 2. Longitudinal acceleration during a single test run involving AB deployment (rider P01, session 2, run 3) 


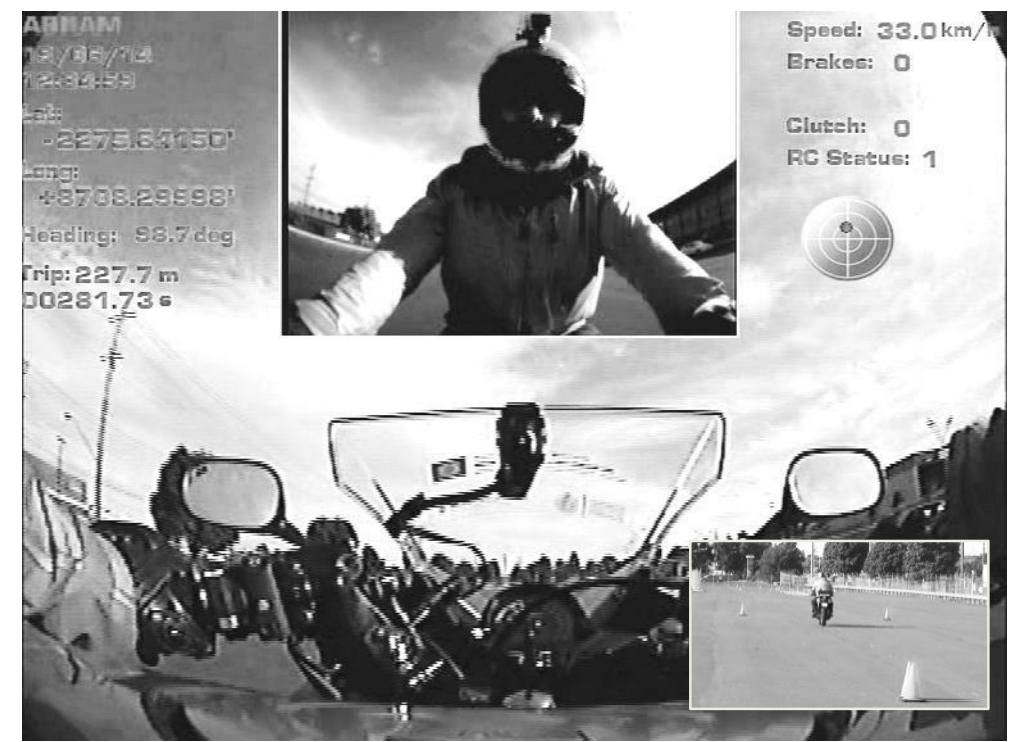

Figure 3. Screenshot from on-board video recording during a test run (inset: screenshot from the video taken with an external video camera)

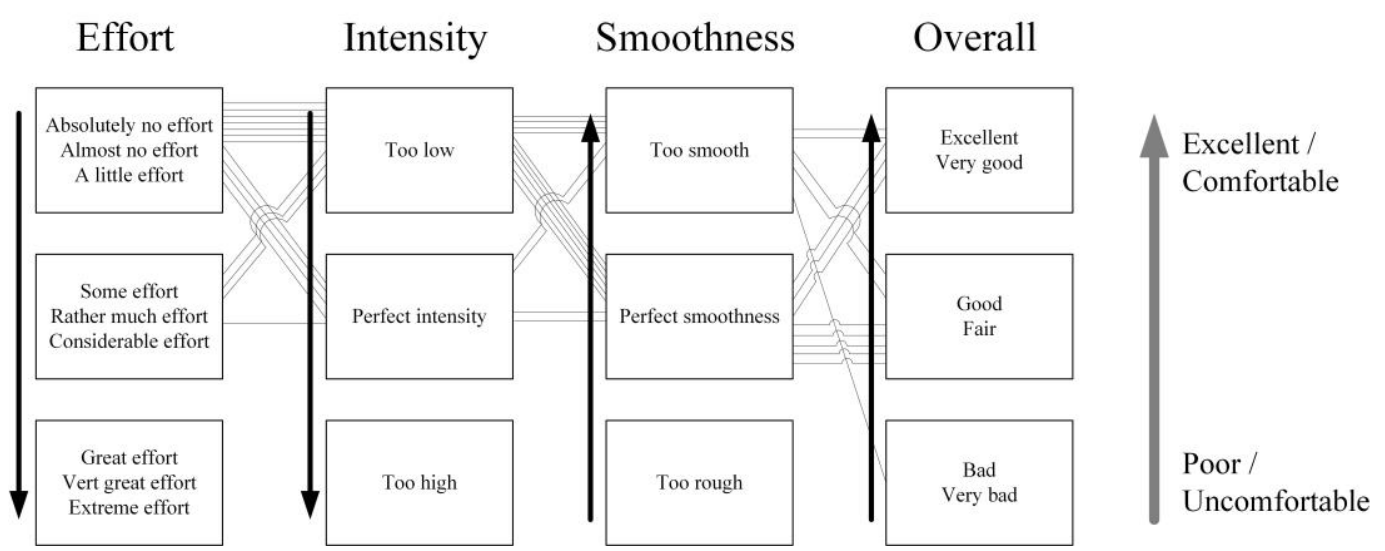

Figure 4. Reported frequencies between response categories with respect to effort, intensity, smoothness and overall rating of the system 